\title{
Aterosclerose Carotídea e Hipodensidades da Substância Branca: uma Relação Controversa
}

\author{
Carotid Atherosclerosis and White Matter Hypodensities: a Controversial \\ Relationship
}

\author{
Ricardo FÉLIX-MORAIS ${ }^{1}$, João SARGENTO-FREITAS² ${ }^{2}$ Fernando SILVA², Gustavo CORDEIRO², César NUNES ${ }^{1}$, Joana \\ RIBEIRO $^{3}$, Miguel CORDEIRO ${ }^{1}$, Cristina MOURA ${ }^{1}$, Luís CUNHA ${ }^{2}$ \\ Acta Med Port 2014 Sep-Oct;27(5):581-586
}

\section{RESUMO}

Introdução: As hipodensidades da substância branca de provável etiologia vascular, são uma causa importante de morbilidade, condicionando deterioração cognitiva. No entanto, numerosas dúvidas persistem quanto à sua fisiopatologia. O objectivo deste estudo é clarificar o papel da aterosclerose carotídea e outros factores de risco vascular no desenvolvimento das hipodensidades da substância branca de provável etiologia vascular.

Material e Métodos: Realizou-se uma avaliação imagiológica, por tomografia computadorizada crânio-encefálica e ecografia carotídea, com menos de um mês de intervalo. Procedeu-se à recolha de informação sobre os factores de risco vascular. Determinámos associações independentes entre hipodensidades da substância branca de provável etiologia vascular, espessura da íntima média carotídea, estenose carotídea ateromatosa e factores de risco vascular.

Resultados: Foram incluídos 472 doentes, idade média de 67,32 (DP: 14.75), 274 (58,1\%) sexo masculino. Os preditores independentes da hipodensidades da substância branca de provável etiologia vascular foram: idade (OR: 1,067, 95\% IC: 1,049 - 1,086, $p<0,001$ ) e a hipertensão (OR: 1,726,95\% IC: 1,097 - 2,715, $p=0,018$ ). Não foi encontrada uma associação entre a espessura da intima média carotídea (OR: 2,613, 95\% IC: 0,886 - 7,708, $p=0,082$ ) ou grau de estenose carotídea (OR: 1,021, 95\% IC: 0,785 - 1,328, $p=0,877$ ) e hipodensidades da substância branca de provável etiologia vascular.

Discussão: Dos diversos factores de risco analisados, apenas a idade e hipertensão se associaram de forma independente às hipodensidades da substância branca de provável etiologia vascular. Não foi encontrada uma relação entre a aterosclerose extracraniana, expressa pela espessura do complexo intima-média ou grau de estenose, com o desenvolvimento de hipodensidades da substância branca de provável etiologia vascular. Sendo a aterosclerose um fenómeno sistémico, estes achados sugerem que as hipodensidades da substância branca de provável etiologia vascular, tenham um mecanismo alternativo ou concorrente à aterosclerose no seu desenvolvimento.

Conclusão: Os dados deste estudo, sugerem que a idade e hipertensão sejam os principais factores de risco no desenvolvimento de hipodensidades da substância branca de provável etiologia vascular. Não foi encontrada uma associação independente entre a aterosclerose carotídea e as hipodensidades da substância branca de provável etiologia vascular.

Palavras-chave: Cerébro; Doença Carotídea.

\section{ABSTRACT}

Introduction: White matter hypodensities of presumed vascular origin, are recognized as an important cause of morbidity with established clinical and cognitive consequences. Nonetheless, many doubts remain on its physiopathology. Our goal is to clarify the potential role of carotid atherosclerosis and other vascular risk factors in the development of white matter hypodensities of presumed vascular origin.

Material and Methods: We included patients that underwent CT brain scan and neurosonologic evaluation within a one-month period. Full assessment of vascular risks factors was performed. We seek to find independent associations between white matter hypodensities of presumed vascular origin, carotid intima-media thickness and vascular risk factors.

Results: 472 patients were included, mean age was 67.32 (SD: 14.75), 274 (58.1\%) were male. The independent predictors of white matter hypodensities of presumed vascular origin were age (OR: $1.067,95 \%$ IC: $1.049-1.086, p<0.001)$ and hypertension (OR: $1.726,95 \%$ IC: $1.097-2.715, p=0.018$ ). No association was found between IMT (OR: $2.613,95 \%$ IC: $0.886-7.708, p=0.082$ ) or carotid artery stenosis (OR: 1.021, 95\% IC: $0.785-1.328, p=0.877$ ) and white matter hypodensities of presumed vascular origin.

Discussion: Only age and hypertension proved to have an independent association with white matter hypodensities of presumed vascular origin. Carotid atherosclerosis, evaluated by IMT and the degree of carotid artery stenosis, showed no association with white matter hypodensities of presumed vascular origin. Since atherosclerosis is a systemic pathology, these results suggest that alternative mechanisms are responsible for the development of white matter hypodensities of presumed vascular origin.

Conclusion: Age and hypertension seem to be the main factors in the development of white matter hypodensities of presumed vascular origin. No association was found between carotid atherosclerosis and white matter hypodensities of presumed vascular origin.

Keywords: Brain; Carotid Artery Disease.

\section{INTRODUÇÃO}

As alterações da substância branca são identificadas nos estudos imagiológicos crânio-encefálicos, em 11-21\% dos adultos com 64 anos e em até $94 \%$ dos adultos com 82 anos. ${ }^{1}$ Numerosos termos têm sido empregues para as designar. Hachinski, em 1987, ${ }^{2}$ introduziu o termo leukoaraiosis (do grego leuko - branco; araiosis - rarefação) para

1. Departamento de Imagem Médica. Centro Hospitalar e Universitário de Coimbra. Coimbra. Portugal.

2. Departamento de Neurologia. Centro Hospitalar e Universitário de Coimbra. Coimbra. Portugal.

3. Faculdade de Medicina da Universidade de Coimbra. Coimbra. Portugal.

Recebido: 24 de Dezembro de 2013 - Aceite: 21 de Abril de 2014 | Copyright @ Ordem dos Médicos 2014 
denominar as alterações da substância branca peri-ventriculares ou profundas, visíveis na TC e/ou RMN. Estas alterações traduzem-se por áreas de hipersinal em T2, densidade protónica (DP), fluid attenuated inversion recovery (FLAIR) e hiposinal em T1, nos estudos por RMN, sendo a ponderação FLAIR a mais sensível para a sua identificação. Nos estudos por TC, traduzem-se por áreas hipodensas de bordos mal definidos. ${ }^{3}$ Outros termos tem sido empregues para as designar, nomeadamente: leucoencefalopatia microcirculatória isquémica, hiperintensidades da substância branca, alterações da substância branca dependentes da idade. Efetivamente a diversidade da nomenclatura existente traduz as dúvidas que persistem na sua fisiopatologia e a sua controversa correlação clinico-radiológica. Recentemente, fruto de um consenso internacional, foi decidido uniformizar a nomenclatura, devendo estas alterações serem referidas como hipodensidades da substância branca de provável etiologia vascular (HSBPEV) nos estudos por tomografia computadorizada ou por hiperintensidades da substância branca nos estudos por ressonância magnética. ${ }^{3}$ Diversos estudos demonstram que as alterações da substância branca estão associadas a declínio cognitivo., ${ }^{4,5}$ Sendo a sua extensão e progressão factores determinantes desta deterioração. Encontram-se também associadas a alterações da marcha; incontinência urinária; depressão e quedas, ${ }^{6}$ e a um risco aumentado de AVC. ${ }^{7}$ Outros estudos identificaram uma associação entre a presença de lesões da substância branca extensas e um aumento da mortalidade. ${ }^{8}$ As alterações da substância branca terão provavelmente um substrato fisiopatológico multifactorial. Sendo certo o aumento da proporção da população idosa, a prevalência quase endémica das HSBPEV nessa faixa etária e a sua associação a um acréscimo de comorbilidades e mortalidade, torna-se crucial identificar os factores de risco que contribuem para o seu desenvolvimento, afim de ser possível desenvolver acções de prevenção primárias e/ ou secundárias. Alguns estudos, sugerem que a aterosclerose possa desempenhar um papel fisiopatológico no aparecimento desta patologia. ${ }^{9} \mathrm{~A}$ espessura da íntima média (EIM) da artéria carotida comum e a presença de placas ateromatosas nos eixos carotídeos, são marcadores validados e amplamente usados para avaliar a aterosclerose extra-craniana, tendo sidos relacionadas com complicações cerebrovasculares e coronárias. ${ }^{10}$ Assim, será interessante avaliar qual a relação e importância da aterosclerose extracraniana no desenvolvimento das alterações da substância branca anteriormente referidas.

Este estudo, tem assim como objectivo, ajudar a esclarecer o contributo diferencial de diversos factores de risco cerebrovasculares, assim como da aterosclerose carotídea no desenvolvimento de HSBPEV.

\section{MATERIAL E MÉTODOS}

Incluíram-se, de forma consecutiva, os doentes submetidos a uma tomografia computadorizada crânio-encefálica e estudo ecográfico carotídeo, com menos de um mês de intervalo, durante o período compreendido entre Janeiro e Agosto do ano 2011, referenciados para estudo neurosonológico no serviço de neurologia do Centro Hospitalar e Universitário de Coimbra. Nestes doentes realizou-se uma investigação dos factores de risco vasculares que apresentavam, nomeadamente idade, hipertensão arterial, dislipidemia, diabetes mellitus, fibrilhação auricular, insuficiência cardíaca congestiva, tabagismo e obesidade. Excluíram-se os doentes que apresentavam patologias com tradução imagiológica cerebral grave tais como tumores e hemorragias intra-cranianas extensas, que limitariam a correcta avaliação das alterações da substância branca. Este estudo foi submetido e aprovado pela comissão de ética do respectivo centro hospitalar.

\section{Avaliação imagiológica - Classificação Age-Related} White Matter Changes (ARWMC): A avaliação imagiológica das alterações da substância branca foi realizada de forma independente por dois neurorradiologistas cegos à informação clínica, com igual experiência. As alterações da substância branca foram classificadas quanto ao seu grau de forma semiquantitativa recorrendo à escala Age-Related White Matter Changes. ${ }^{11}$ Segundo esta escala, 0 corresponde à ausência de alterações da substância branca, 1

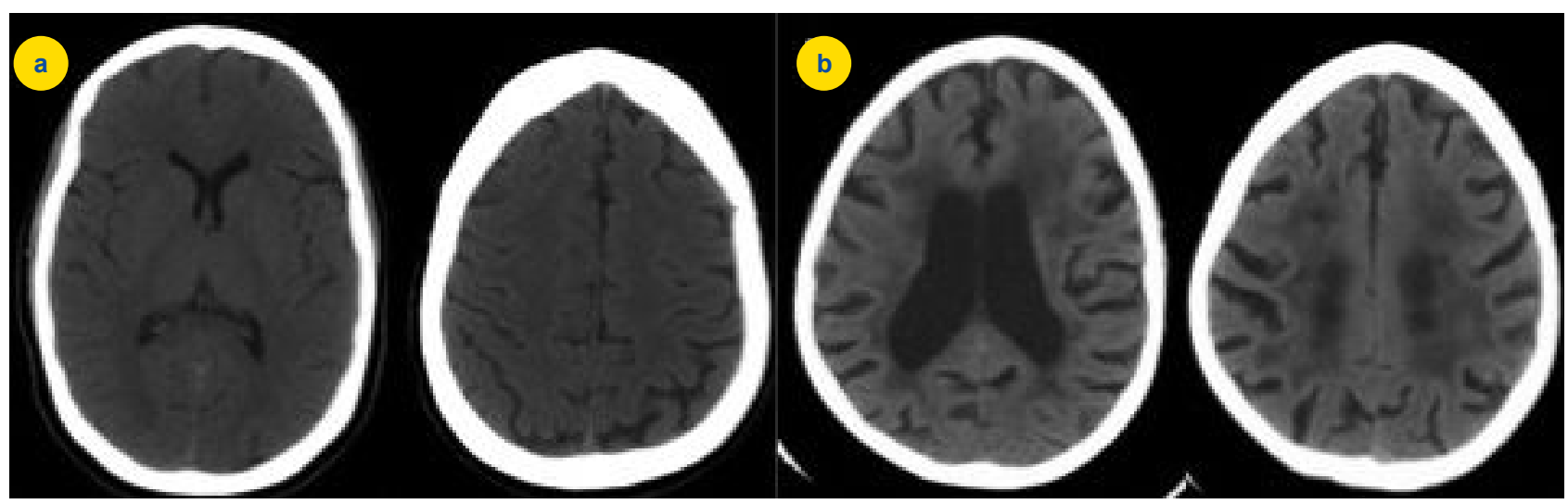

Figura 1 - Avaliação pela escala ARWMC. a) - TC CE cortes axiais: observa-se ausência de alterações da substância branca, grau 0 da escala ARWMC. b) - TC CE cortex axiais: observam-se alterações da susbtância branca periventricular com extensão à substância branca profunda e subcortical, grau 3 ARWMC. 
- alterações focais, 2 - lesões parcialmente confluentes, 3 lesões confluentes. Quando o grau atribuido foi discrepante entre os dois neurorradiologistas, o score final foi decidido por consenso. As hipodensidades da substância branca a nível dos gânglios da base e do tronco cerebral não foram incluídas na avaliação, de acordo com as novas regras de consenso. ${ }^{3}$ As lesões vasculares do tipo lacunar foram excluídas, tendo estas sido definidas como lesões de bordos bem delimitados, morfologia arredondada, diâmetro 3-15 $\mathrm{mm}$, com densidade idêntica à do liquido cefalorraquidiano. ${ }^{3,11}$

Todos os estudos tomodensitométricos cranioencefálicos, foram efectuados num equipamento de tomografia computadorizada GE Light Speed, 64 detectores, com os seguintes parâmetros técnicos: matriz 512 x 512, SFOV 25 cm, CTDlvol 76 mGy, 330 mAs, 120 kv. A espessura de corte foi de $2,5 \mathrm{~mm}$ para região infra-tentorial a $5 \mathrm{~mm}$ para a região supra-tentorial.

Ecografia carotídea: A avaliação hemodinâmica foi realizada por dois neurossonologistas de igual experiência, utilizando um ecógrafo General Electric ${ }^{\circledR}$ modelo Logiq 7, com uma sonda linear de 7,5 MHz para a avaliação extra-craniana. Cada doente foi estudado por um de dois neurossonologistas com idêntica experiência. A obtenção das imagens foi realizada com o doente em decúbito dorsal, com o pescoço ligeiramente estendido e a cabeça rodada contralateralmente ao lado examinado. A espessura da intima média foi medida na artéria carótida comum, a 10 mm proximal à sua bifurcação, recorrendo a ângulos de insonação ideais. ${ }^{10} \mathrm{~A}$ espessura da intima média (EIM) foi medida por método manual em ambas as artérias carótidas comuns e a média destas usada na análise estatística. A quantificação da estenose carotídea ateromatosa foi realizada no início da artéria carótida interna $(\mathrm{ACl})$ de acordo com os critérios de Grant 2003. ${ }^{10} \mathrm{As} \mathrm{ACl}$ de todos os doentes foram classificadas bilateralmente como: normais (sem evidência de qualquer placa ateromatosa), estenose $<50 \%$ (presença de placas ateromatosas sem significado hemo- dinâmico), estenose $50-69 \%$, estenose $>70 \%$ e inferior a suboclusão, suboclusão e oclusão. Para análise estatística utilizámos o maior grau de estenose observado numa $\mathrm{ACl}$ para cada doente (por exemplo: num doente com patologia ateromatosa carotídea condicionando estenose $<50 \%$ na $\mathrm{ACl}$ esquerda e estenose $50-69 \%$ na $\mathrm{ACl}$ direita foi considerado este último grau de estenose). A presença de patologia não ateromatosa condicionando estenose/oclusão carotídea (como dissecção, vasculite ou êmbolo cardíaco) não foi considerada para análise.

\section{Factores de risco vasculares}

Os factores de risco vascular considerados neste estudo, são: idade, hipertensão arterial (HTA), dislipidémia, diabetes mellitus (DM) tipo 1 e 2 (para análise estatística as formas tipo 1 e 2 foram analisadas em conjunto), obesidade (definida como índice de massa corporal > 30), insuficiência cardíaca congestiva (ICC), fibrilhação auricular (FA) e tabagismo (activo ou antigo); foram identificados por questionário ao médico assistente que requereu o exame imagiológico e confirmados por consulta dos registos clínicos.

\section{Análise estatística}

A análise estatística foi realizada recorrendo ao software SPSS Statistics, versão 17. Realizámos um estudo seccional cruzado de todos os doentes incluídos. Após caracterização da amostra em estudo, incluindo a média (desvio padrão) para as variáveis continuas e número absoluto (percentagem) para as variáveis categóricas, efectuámos dois modelos de regressão ordinal multivariável incluindo todos os factores de risco vascular estudados e espessura da intima média ou grau de estenose carotídea ateromatosa. Atendendo à elevada dimensão da amostra, ao número de variáveis em estudo e objetivos do trabalho optámos por incluir todas as variáveis estudadas nos modelos de regressão. Assumimos significado estatístico para $p<0,05$.
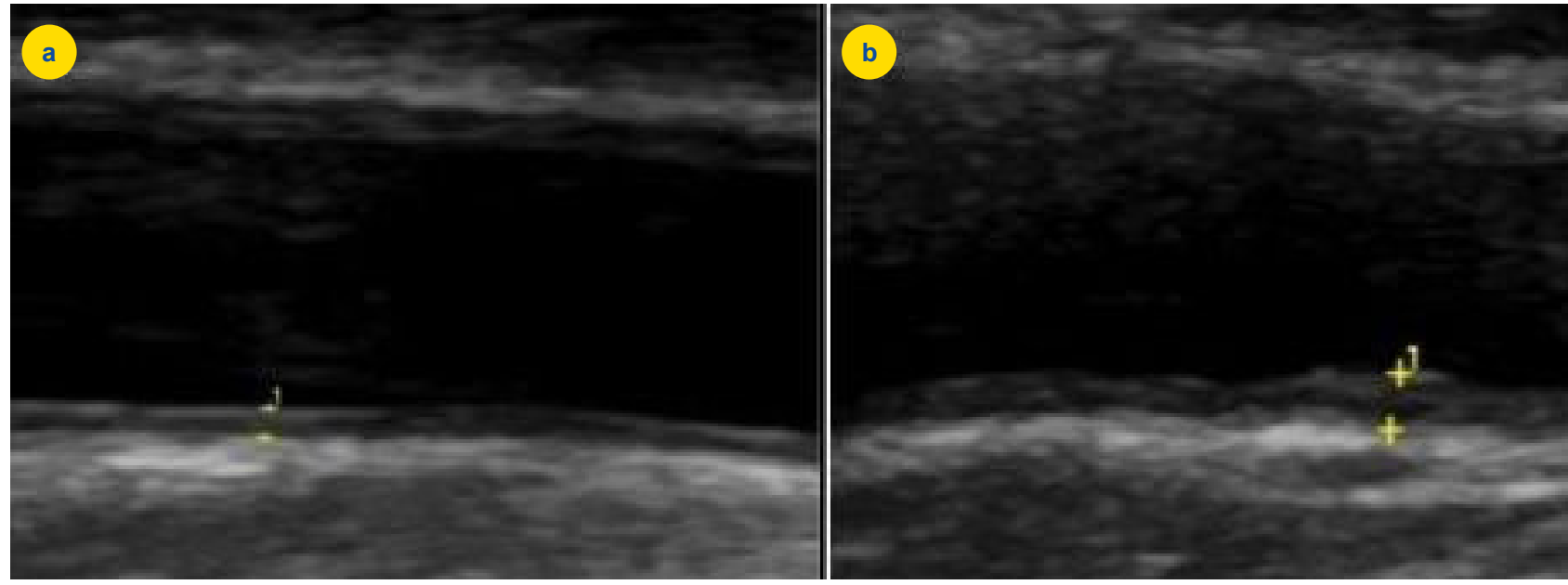

Figura 2 - Medição da EIM. Medição por método manual em ambas as artérias carótidas comuns (a e b), observando-se aumento da EIM em b. 


\section{RESULTADOS}

\section{População}

Foram incluídos um total de 472 doentes. A caracterização demográfica da população em estudo, assim como a prevalência dos factores de risco vascular e a medição da espessura da intima média, é apresentada na tabela 1.

\section{Factores de risco}

Na tabela 2 expressam-se os resultados da regressão ordinal para identificação de factores preditores independentes das HSBPEV, incluindo-se na análise todos os factores de risco vascular do estudo e o grau máximo de estenose carotídeo.
$\mathrm{Na}$ tabela 3 expressam-se os resultados da regressão ordinal para identificação de factores preditores independentes das HSBPEV, incluindo-se na análise todos os factores de risco vascular do estudo e a espessura da íntima média da artéria carotídea.

$\mathrm{Na}$ análise efectuada entre os factores de risco vascular e HSBPEV, destaca-se a forte associação independente entre HSVPEV com a idade (OR: 1,072, IC 95\%: 1,054$1,090, p<0,001)$ e hipertensão arterial (OR: 1,797, IC 95\%: $1,163-7,776, p=0,008)$. Não foi encontrada uma associação independente entre a espessura da intima média da artéria carotídea ou a estenose carotídea e as HSBPEV.

Tabela 1 - Caracterização dos factores de risco vascular e da espessura da intima media carotídea na população em estudo

\begin{tabular}{lccc}
\hline Variável & $\begin{array}{c}\text { Total população } \\
(\boldsymbol{n}=\mathbf{4 7 2 )}\end{array}$ & $\begin{array}{c}\text { Sem hipodensidades da } \\
\text { substância branca } \\
(\boldsymbol{n}=160)\end{array}$ & $\begin{array}{c}\text { Com hipodensidades da } \\
\text { substância branca } \\
(\boldsymbol{n}=\mathbf{3 1 2})\end{array}$ \\
\hline Idade & $67,32(14,75)$ & $58,33(15,16)$ & $71,93(12,21)$ \\
Sexo masculino & $274(58,1 \%)$ & $103(64,4 \%)$ & $171(54,8 \%)$ \\
HTA & $331(70,1 \%)$ & $90(56,3 \%)$ & $241(77,2 \%)$ \\
DM & $112(23,7 \%)$ & $27(16,9 \%)$ & $85(27,2 \%)$ \\
Dislipidemia & $219(46,4 \%)$ & $72(45,0 \%)$ & $147(47,1 \%)$ \\
Tabagismo & $42(8,9 \%)$ & $21(13,1 \%)$ & $21(6,7 \%)$ \\
FA & $88(18,6 \%)$ & $15(9,4 \%)$ & $73(23,4 \%)$ \\
Obesidade & $41(8,7 \%)$ & $11(6,9 \%)$ & $30(9,6 \%)$ \\
ICC & $46(9,7 \%)$ & $10(6,3 \%)$ & $36(11,5 \%)$ \\
EIM & $0,79(0,19)$ & $0,71(0,20)$ & $0,83(0,19)$ \\
Presença de placas carotídeas & $179(37,9 \%)$ & $78(48,8 \%)$ & $254(81,4 \%)$ \\
\hline
\end{tabular}

HTA: Hipertensão arterial; FA: Fibrilhação auricular; DM: Diabetes Mellitus; ICC: Insuficiência cardiaca congestiva; EIM - Espessura da intima média da artéria carótida comum.

Tabela 2 - Regressão ordinal para identificação de factores preditores independentes das hipodensidades da substância branca, incluindo os diversos factores de risco vascular e o maior grau de estenose carotídea

\begin{tabular}{lcccc}
\hline Variável & OR & \multicolumn{2}{c}{ IC $\mathbf{9 5} \%$} & $\boldsymbol{p}$ \\
\hline Estenose carotídea & 1,021 & 0,785 & 1,328 & 0,877 \\
Tabagismo & 0,724 & 0,368 & 1,423 & 0,349 \\
FA & 1,142 & 0,707 & 1,846 & 0,587 \\
ICC & 1,260 & 0,681 & 2,329 & 0,462 \\
Idade & $\mathbf{1 , 0 7 2}$ & $\mathbf{1 , 0 5 4}$ & $\mathbf{1 , 0 9 0}$ & $<\mathbf{0 , 0 0 1}$ \\
HTA & $\mathbf{1 , 7 9 7}$ & $\mathbf{1 , 1 6 3}$ & $\mathbf{2 , 7 7 6}$ & $\mathbf{0 , 0 0 8}$ \\
Dislipidemia & 0,941 & 0,651 & 1,359 & 0,745 \\
Obesidade & 1,277 & 0,702 & 2,323 & 0,424 \\
DM & 1,190 & 0,778 & 1,818 & 0,422 \\
Sexo masculino & 1,125 & 0,771 & 1,642 & 0,540 \\
\hline
\end{tabular}

HTA: Hipertensão arterial; FA: Fibrilhação auricular; DM: Diabetes Mellitus; ICC: Insuficiência cardíaca congestiva. 
Tabela 3 - Regressão ordinal para identificação de factores preditores independentes das hipodensidades da substância branca, incluindo os diversos factores de risco vascular e a espessura da intima média da artéria carótida comum

\begin{tabular}{|c|c|c|c|c|}
\hline \multirow{2}{*}{$\begin{array}{l}\text { Variável } \\
\text { EIM }\end{array}$} & \multirow{2}{*}{$\begin{array}{c}\text { OR } \\
2,613\end{array}$} & \multicolumn{2}{|c|}{ IC $95 \%$} & \multirow{2}{*}{$\begin{array}{c}p \\
0,082\end{array}$} \\
\hline & & 0,886 & 7,708 & \\
\hline Tabagismo & 0,635 & 0,315 & 1,282 & 0,635 \\
\hline FA & 1,099 & 0,667 & 1,809 & 0,712 \\
\hline ICC & 1,307 & 0,692 & 2,467 & 0,409 \\
\hline Idade & 1,067 & 1,049 & 1,086 & $<0,001$ \\
\hline HTA & 1,726 & 1,097 & 2,715 & 0,018 \\
\hline Dislipidemia & 0,907 & 0,624 & 1,319 & 0,610 \\
\hline Obesidade & 1,405 & 0,727 & 2,714 & 0,311 \\
\hline DM & 1,190 & 0,771 & 1,834 & 0,432 \\
\hline Sexo masculino & 1,141 & 0,770 & 1,692 & 0,510 \\
\hline
\end{tabular}

\section{DISCUSSÃO}

A associação entre HSBPEV e factores de risco cerebrovascular, tem sido inconstante nos diversos estudos existentes na literatura. ${ }^{12,13}$ No nosso estudo, não foi encontrada uma associação entre HSBPEV e a dislipidemia, tabagismo, fibrilhação auricular, obesidade, diabetes mellitus e insuficiência cardíaca congestiva.

A caracterização da amostra (Tabela 1), revela que os doentes com maior grau de HSBPEV têm igualmente uma maior estenose carotídea e maior espessura da intima média. Contudo na análise multivariada (Tabelas 2 e 3) verificamos que a aterosclerose carotídea não tem uma correlação estatisticamente significativa com as HSBPEV. Sugerindo então que a aterosclerose carotídea, esteja efectivamente mais presente no grupo dos indivíduos com mais idade e patologia hipertensiva, mas não sendo per se um factor de risco no desenvolvimento de HSBPEV. Atendendo ao facto do processo aterosclerótico ser sistémico ${ }^{14}$ a ausência de uma correlação entre as HSBPEV e aterosclerose extra-craniana, neste estudo aferida pelo grau de estenose carotídea e EIM, um dos marcadores mais usados na literatura, ${ }^{10}$ sugerem eventuais mecanismos alternativos ou concorrentes à ateromatose no seu desenvolvimento. Um desses possíveis mecanismos recentemente proposto no âmbito de uma revisão alargada da literatura por sociedades envolvidas no estudo das HSBPEV, ${ }^{3,15}$ é o da disrupção da barreira hemato-encefálica (BHE) por um processo secundário à disfunção endotelial dos vasos endocranianos de pequeno calibre. ${ }^{14-16}$

\section{Analisando as associações positivas}

Hipertensão: Tanto a hipertensão sistólica como diastólica têm sido associados a alterações da substância branca. ${ }^{17,18} \mathrm{~A}$ hipertensão arterial induz, nas artérias de pequeno calibre, processos de degeneração fibrinoide e lipohialinose da túnica média, o que contribui para o estreitamento e eventual obliteração do lúmen das pequenas artérias e arteríolas perfurantes que suprem a substância branca profunda/subcortical..$^{15}$ As artérias perfurantes possuem um sistema anastomótico relativamente pobre, tornando assim a substância branca na sua dependência vulnerável à lesão destes vasos. A hipertensão induz também alterações da permeabilidade da BHE e por este motivo pode causar lesões da substância branca por edema, destruição enzimática, activação astrocitária ${ }^{15,19}$ sendo, por isso considerada, um factor de risco directo na indução da disfunção endotelial. Estes fenómenos, poderão estar na base da associação positiva encontrada neste estudo entre a presença hipertensão arterial e as HSBPEV. No entanto, é de salientar que novos estudos têm demonstrado existir uma base genética na associação entre a hipertensão e as alterações da substância branca. ${ }^{20,21}$. Podendo esta ser uma das explicações pelos resultados menos satisfatórios que o antecipado no uso de anti-hipertensores para prevenir as HSBPEV. ${ }^{15}$

Idade: As alterações da substância branca mais frequentes em pessoas idosas, são as de grau 1 da escala ARWMC e encontram-se presentes em mais de metade dos doentes assintomáticos com mais de 55 anos $^{22}$ Tornando-se endémicas em população mais idosas, com uma prevalência de cerca de $92 \%,{ }^{1,13}$ razão pela qual estas alterações são por vezes apelidadas de alterações da substância branca dependentes da idade. A permeabilidade do endotélio cerebrovascular aumenta com a idade, de forma exponencial; ${ }^{23}$ como em muitos dos eventos relacionados com a idade, a perda da integridade endotelial cerebrovascular, pode iniciar-se em diferentes idades e progredir de forma diferentes, no entanto a idade é um dos seus factores predictores mais importantes na indução da disfunção endotelial. É também interessante notar-se que tem sido demonstrado aumento da permeabilidade da $\mathrm{BHE}^{23} \mathrm{em}$ 
doentes com demência de Alzheimer.

\section{Limitações do estudo}

Este estudo apresenta diversas limitações, tal como o possível viés de selecção da amostra, pelo facto de ser tratar de um estudo monocêntrico mas também pelos doentes terem sido incluídos baseando-se na sua necessidade de cuidados médicos e avaliação neuroimagiológica. A duração e severidade dos factores de risco, assim como terapêuticas que visam controlar estes factores de risco não foram tidos em conta neste estudo. Esta opção deveu-se à dificuldade de obter, de forma fidedigna, estes dados de forma retrospectiva. O recurso à TC CE para avaliação do grau de HSBPEV, poderá ser visto como uma limitação, no entanto a avaliação qualitativa realizada com recurso a escalas neuroimagiológicas, conferem lhe uma maior validade, sendo que a principal vantagem da RMN se traduz essencialmente numa maior detecção das lesões de menor grau, mais importante em análises quantitativas. ${ }^{11}$

\section{REFERÊNCIAS}

1. American Psychiatric Association. Diagnostic and statistical manual of mental disorders. $4^{\text {th }}$ ed. Chicago: APA; 1994.

2. Hachinski VC, Potter P, Merskey H. Leuko-araiosis. Arch Neurol. 1987;44:21-3

3. Wardlaw JM, Smith EE, Biessels GJ, Cordonnier C, Fazekas F, Frayne $\mathrm{R}$, et al. Neuroimaging standards for research into small vessel disease and its contribution to ageing and neurodegeneration. Lancet Neurol. 2013;12:822-38.

4. Mok VC, Wong A, Lam WW, Fan YH, Tang WK, Kwok T, et al. Cognitive impairment and functional outcome after stroke associated with small vessel disease. J Neurol Neurosurg Psychiatry. 2004;75:560-6.

5. van der Flier WM, Van Straaten EC, Barkhof F, Verdelho A, Madureira S, Pantoni S, et al. Small vessel disease and general cognitive function in nondisabled elderly: the LADIS study. Stroke. 2005;36:2116-20.

6. van der Flier WM, van Straaten EC, Barkhof F, Verdelho A, Madureira S Pantoni L, et al. Cerebral white matter lesions, gait, and the risk of incident falls: a prospective population-based study. Stroke. 2009;40:175-180.

7. Buyck JF, Dufouil $C$, Mazoyer $B$, Maillard $P$, Ducimetière $P$, Alpérovitch $A$, et al. Cerebral white matter lesions are associated with the risk of stroke but not with other vascular events: the 3-city dijon study. Stroke. 2009;40:2327-31.

8. Oksala NK, Oksala A, Pohjasvaara T, Vataja R, Kaste M, Karhunen PJ et al. Age related white matter changes predict stroke death in long term follow-up. J Neurol Neurosurg Psychiatry. 2009;80:762-6.

9. Jong G, Kessels F, Lodder J. Two types of lacunar infarcts: further arguments from a study on prognosis. Stroke. 2002;33:2072-6.

10. Grant EG, Benson CB, Moneta GL, Alexandrov AV, Baker JD, Bluth El, et al. Carotid artery stenosis: gray-scale and Doppler US diagnosis - Society of Radiologists in Ultrasound Consensus Conference. Radiology. 2003;229:340-6.

11. Wahlund LO, Barkhof F, Fazekas F, Bronge L, Augustin M, Sjögren M, et al. European Task Force on Age-Related White Matter Changes. A new rating scale for age-related white matter changes applicable to MRI and CT. Stroke. 2001;32:1318-22.

12. Gouw AA, van der Flier WM, Pantoni $L$, Inzitari $D$, Erkinjuntti $T$, Wahlund $L O$, et al. On the etiology of incident brain lacunes: longitudinal

\section{CONCLUSÕES}

Os resultados deste estudo sugerem que as HSBPEV tenham a idade e hipertensão como substrato fisiopatológico principal. Não foi encontrada uma associação independente entre a aterosclerose carotídea e as HSBPEV, sugerindo que este não seja o seu principal factor etiológico, no entanto mais estudos são necessários para avaliar a repercussão das alterações macrocirculatórias na etiologia das HSBPEV.

\section{CONFLITOS DE INTERESSE}

Os autores declararam a inexistência de quaisquer conflitos de interesse.

\section{FONTES DE FINANCIAMENTO}

Os autores declararam a inexistência de quaisquer fontes de financiamento externas.

observations from the LADIS study. Stroke. 2008;39:3083-5

13. de Leeuw FE, de Groot JC, Achten E, Oudkerk M, Ramos LM, Heijboer $\mathrm{R}$, et al. Prevalence of cerebral white matter lesions in elderly people: a population based magnetic resonance imaging study. The Rotterdam Scan Study. J Neurol Neurosurg Psychiatry. 2001;70:9-14.

14. Stevenson SF, Doubal FN, Shuler K, Wardlaw JM. A systematic review of dynamic cerebral and peripheral endothelial function in lacunar stroke versus controls. Stroke. 2010;41:434-42.

15. Wardlaw JM, Smith C, Dichgans M. Mechanisms of sporadic cerebral small vessel disease: insights from neuroimaging. Lancet Neurol. 2013;12:483-97.

16. Fazekas F, Ropele S, Enzinger C, Gorani F, Seewann A, Petrovic K, et al. MTI of white matter hyperintensities. Brain. 2005;128:2926-32.

17. Longstreth WT Jr, Manolio TA, Arnold A, Burke GL, Bryan N, Jungreis $\mathrm{CA}$, et al. Clinical correlates of white matter findings on cranial magnetic resonance imaging of 3301 elderly people: the Cardiovascular Health Study. Stroke. 1996;27:1274-82.

18. Breteler MM, van Swieten JC, Bots ML, Grobbee DE, Claus JJ, van den Hout $\mathrm{JH}$, et al. Cerebral white matter lesions, vascular risk factors, and cognitive function in a population-based study: the Rotterdam Study. Neurology. 1994;44:1246-52.

19. Schmidt R, Schmidt H, Haybaeck J, Loitfelder M, Weis S, Cavalier $M$, et al. Heterogeneity in age-related white matter changes. Acta Neuropathol. 2011;122:171-85.

20. Kochunov P, Glahn D, Lancaster J, Winkler A, Kent JW Jr, Olvera RL, et al. Whole brain and regional hyperintense white matter volume and blood pressure: overlap of genetic loci produced by bivariate, wholegenome linkage analyses. Stroke. 2010;41:2137-42.

21. Kochunov P, Glahn DC, Lancaster J, Winkler A, Karlsgodt K, Olvera RL, et al. Blood pressure and cerebral white matter share common genetic factors in Mexican Americans. Hypertension. 2011;57:330-5.

22. Xiong YY, Mok V. Age-related white matter changes. J Aging Res. 2011;2011:617927.

23. Farrall AJ, Wardlaw JM. Blood brain barrier: ageing and microvascular disease: systemic review and meta-analysis. Neurobiol Aging. 2007;30:337-52. 
Ricardo FÉLIX-MORAIS, João SARGENTO-FREITAS, Fernando SILVA, Gustavo CORDEIRO, César NUNES, Joana RIBEIRO, Miguel CORDEIRO, Cristina MOURA, Luís CUNHA

\section{Aterosclerose Carotídea e Hipodensidades da Substância Branca: uma Relação Controversa}

Acta Med Port 2014:27:581-586

Publicado pela Acta Médica Portuguesa, a Revista Científica da Ordem dos Médicos

Av. Almirante Gago Coutinho, 151

1749-084 Lisboa, Portugal.

Tel: +351218428 215

E-mail: submissao@actamedicaportuguesa.com

www.actamedicaportuguesa.com

ISSN:0870-399X | e-ISSN: 1646-0758

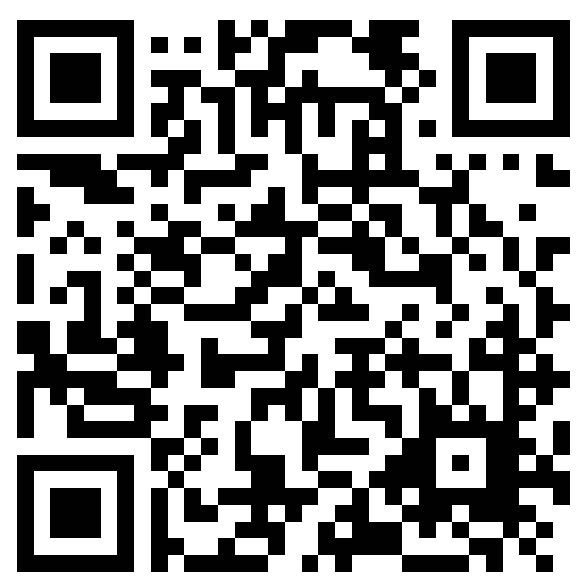

